\title{
Developing a Voltage-Stability-Constrained Security Assessment System Part I : Determination of Power System Voltage Security Operation Limits
}

\author{
N. C. Chang, J. F. Su, Z. B. Du, L. B. Shi, H. F. Zhou, Peter T. C. Tam, Y. X. Ni, Felix F. Wu*
}

\begin{abstract}
The method for determining the voltage security operation limits in a practical voltage security analysis (VSA) system based on VSAT software for large power systems is introduced in this paper. These operation limits include bus voltage limits, branch/corridor transfer power limits and P-load limit of the whole system. The voltage security operation limits are determined by the most critical contingency among the studied contingency set. The most critical contingency determines the P-load limit of the whole system, and all kinds of operation parameter limits are operation parameter values corresponding to this P-load limit under pre-contingency. An operation parameter limit is upper limit if the function relationship between this operation parameter and load power is an increasing curve, or lower limit if the function relationship between this operation parameter and load power is an decreasing curve. These operation parameter limits are helpful for operators to monitor the system operation state.
\end{abstract}

Index Terms-voltage security analysis, operation limits, power systems

\section{INTRODUCTION}

$\mathrm{T}$ HE market mechanism is introducing into power industry around the world. Power markets can improve the efficiency of power industry, but at the same time it will push power system operation state closer to its limit, which makes power system security analysis and monitoring system more and more important $[1,2]$. This paper focuses on power system voltage security analysis.

This is the first part in a two-part paper on the development of a voltage-stability-constrained security assessment system (VSC-SAS), with emphasis on fundamental physical concept and how to determine system operation limits mainly about bus voltage and line or corridor transfer power with voltage-stable-constraints and system security considered. The main aim of the developed VSC-SAS is to provide system operation limits with respect to a usual contingency set. These operation limits include bus voltage limits, branch/corridor transfer power limits, active power loading limit of the whole system etc. In practical operation, real-time operation conditions from SCADA/EMS can be compared with these limits and the distance between them represents power system voltage-security-constrained margin.

* The authors are with the department of Electrical and Electronic, the Univ. of Hong Kong, Hong Kong SAR (e-mail: ncchang@eee.hku.hk)
The operation limits gotten by VSC-SAS can be visualized for operators to monitor power system operation state and know how far the current operation state is from voltage stability boundary. It is obvious that this kind of information is significant for the operator to avoid voltage instability and collapse. In addition to VSC, line power thermal limits and generator capacity limits etc. should also be considered. The security criteria can be $\mathrm{N}-1$ or possible $\mathrm{N}-2$ contingencies. The fundamental physical concept and calculation in determination of system operation limits is the key issue, which is presented below.

The overall structure of the developed VSC-SAS is as the following figure.

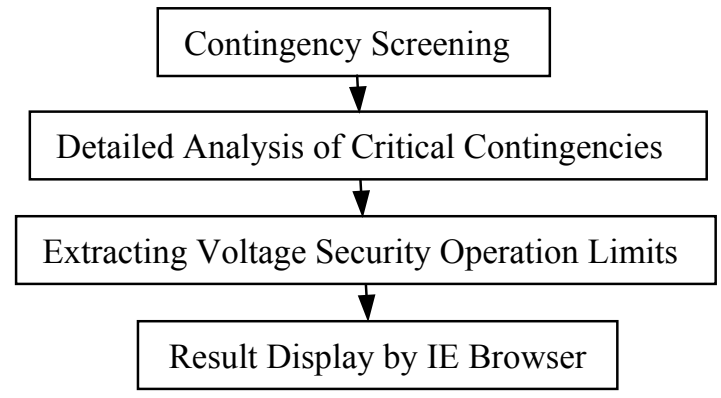

Fig. 1 Overall structure of developed VSA system

\section{Bus Voltage Limits}

Suppose that there are two contingency $A_{1}, A_{2}$, Fig. 2 shows PV-curves of bus $j$ under pre-contingency (base case), contingency $A_{1}$, and contingency $A_{2} . C_{1}$ is the nose point of $\mathrm{P}-\mathrm{V}$ curve for contingency $A_{1}$. Contingency $A_{1}$ includes load shedding protection scheme while contingency $A_{2}$ doesn't. Note that the starting point of $\mathrm{P}-\mathrm{V}$ curve for contingency $A_{1}$ is not the same as that of base case because of load shedding. With the increasing of P-load the operation point under base case moves towards $T_{1}$. As shown in Fig. 2, $T_{1}$ becomes $C_{1}$ under contingency $A_{1}$, and $C_{2}$ under contingency $A_{2}$. It is easy to see that if the operation point beyond $T_{1}$ under base case it is not secure since if contingency $A_{1}$ occurs the postcontingency operation point doesn't exist. Contingency $A_{1}$ is 


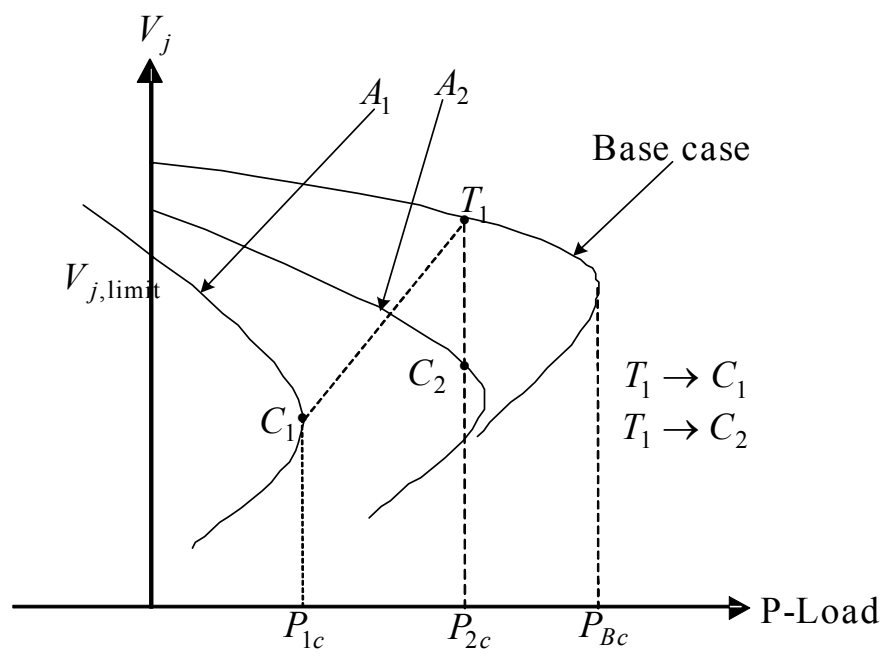

Fig. 2 Bus voltage limit determined by Contingency $A_{1}$

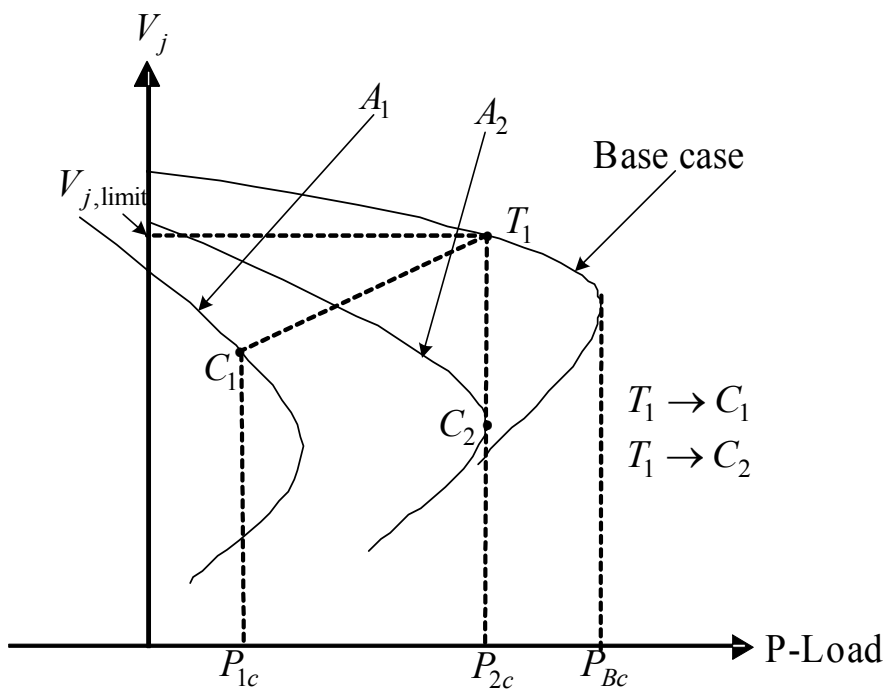

Fig. 3 Bus voltage limit determined by Contingency $\mathrm{A}_{2}$

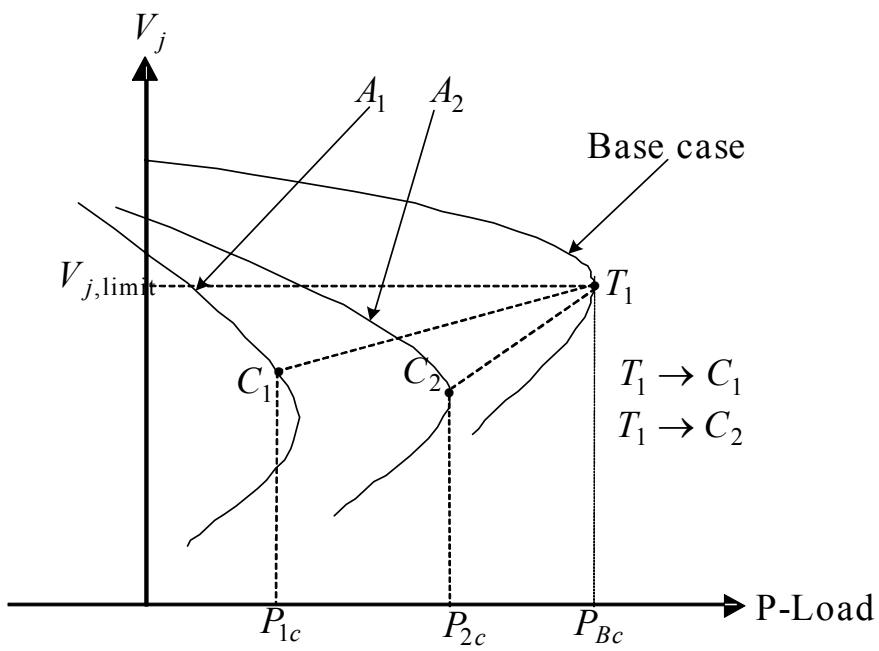

Fig. 4 Bus voltage limit determined by base case

more critical than contingency $A_{2}$. The voltage limit of bus

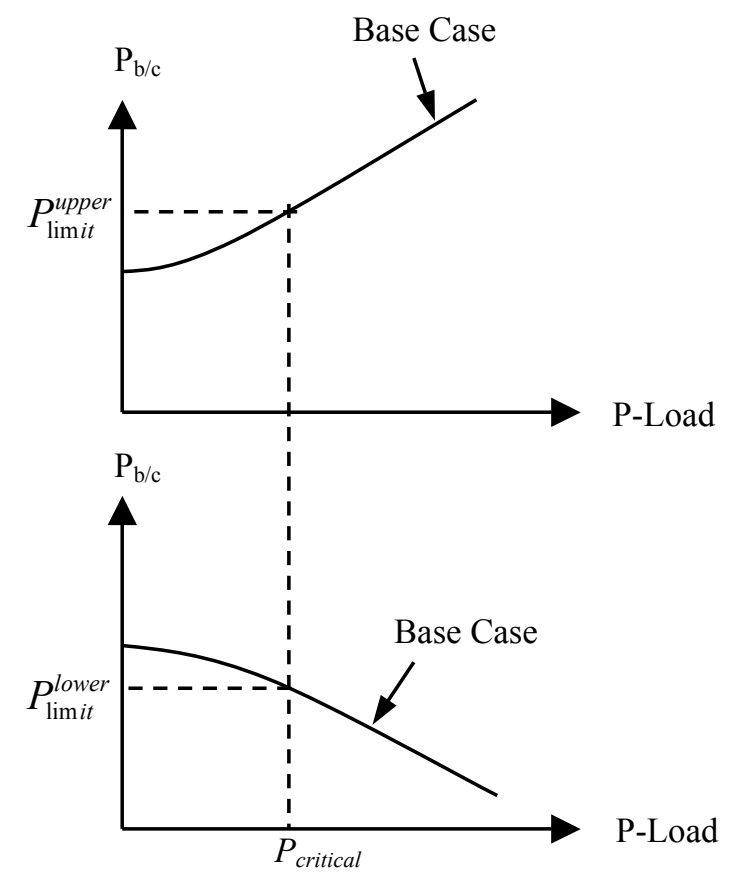

Fig. 5 Branch/corridor transfer power limits

$j$ is the voltage value corresponding to $T_{1}$ under base case, which is determined by contingency $A_{1}$. We call P-load value of $T_{1}$ critical power.

In the above case, the voltage limit of bus $j$ is determined by contingency $A_{1}$. Fig. 3 shows the situation where the voltage limit of bus $j$ is determined by contingency $A_{2}$. Fig. 4 shows the situation where the voltage limit of bus $j$ is determined by base case.

\section{BRANCH/CORRIDOR TRANSFER POWER LIMITS}

On the curve branch/corridor transfer power - load power of branch/corridor $j$ (PP curve), the transfer power limit for branch/corridor $j$ is the branch/corridor transfer power value corresponding to the critical load power $P_{\text {critical }}$, See Fig. 5.

Note that if the PP curve of branch/corridor $j$ is an increasing curve the branch/corridor transfer power limit $P_{j, \lim i t}$ we find is upper limit

$$
P_{j, \lim i t}=P_{\lim i t}^{u p p e r}
$$

That is, $P_{j}<P_{j, \text { limit }}$ is considered as safe operating state under this situation. If the PP curve of branch/corridor $j$ is a decreasing curve, the branch/corridor transfer power limit $P_{j, \lim i t}$ we find is lower limit

$$
P_{j, \lim i t}=P_{\lim i t}^{\text {lower }}
$$

That is, $P_{j}>P_{j, \lim i t}$ is considered as safe operating state under this situation. 


\section{MATHEMATICAL Description OF PROCEDURE TO FIND} ALL LIMITS

Suppose that the direction of load increasing is $\vec{d}$ (increasing step vector), the initial value of load vector is $\boldsymbol{P}_{0}$, load vector $\boldsymbol{P}$ can be written as

$$
\boldsymbol{P}=\boldsymbol{P}_{0}+k \cdot \overrightarrow{\boldsymbol{d}}, \quad k=0,1,2, \cdots
$$

The load flow equation for contingency $C_{i}$ can be written as

$$
\boldsymbol{F}\left(\boldsymbol{P}_{0}+k \cdot \overrightarrow{\boldsymbol{d}}, \boldsymbol{V}_{k}^{i}, C_{i}\right)=0, \quad i=0,1,2, \cdots, N
$$

And so there are some inequality constraints such as maximum generation capacity, branch thermal capacity etc

$$
\boldsymbol{G}\left(\boldsymbol{P}_{0}+k \cdot \overrightarrow{\boldsymbol{d}}, \boldsymbol{V}_{k}^{i}, C_{i}\right) \leq 0, \quad i=0,1,2, \cdots, N
$$

where $i=0$ represents pre-contingency (base case), i.e. with no contingency occurring. $\boldsymbol{V}_{k}^{i}$ is the voltage vector after contingency $C_{i}$ occurs at the load vector value $\boldsymbol{P}_{0}+k \cdot \overrightarrow{\boldsymbol{d}}$.

Definition 1: Critical Power $\boldsymbol{P}_{c r}$ for voltage stability is defined as

$$
\boldsymbol{P}_{c r}=\boldsymbol{P}_{0}+K \cdot \overrightarrow{\boldsymbol{d}}
$$

where $K$ is an integer satisfying that

i) For $\forall k \leq K, \forall i \leq N, \boldsymbol{F}\left(\boldsymbol{P}_{0}+k \cdot h, \boldsymbol{V}_{k}^{i}, C_{i}\right)=0$ is solvable and $\boldsymbol{G}\left(\boldsymbol{P}_{0}+k \cdot \overrightarrow{\boldsymbol{d}}, \boldsymbol{V}_{k}^{i}, C_{i}\right) \leq 0$ is also satisfied;

ii) For $k=K+1$, there at least exist one integer $I$ such that $\boldsymbol{F}\left(\boldsymbol{P}_{0}+k \cdot h, \boldsymbol{V}_{k}^{I}, C_{I}\right)=0$ cannot be solvable or $\boldsymbol{G}\left(\boldsymbol{P}_{0}+k \cdot \overrightarrow{\boldsymbol{d}}, \boldsymbol{V}_{k}^{i}, C_{i}\right) \leq 0$ is violated.

Definition 2: Voltage limit $\boldsymbol{V}_{\lim i t}$ is defined as the solution of $\boldsymbol{F}\left(\boldsymbol{P}_{c r}, \boldsymbol{V}_{K}^{0}, C_{0}\right)=0$, i.e.

$$
\boldsymbol{F}\left(\boldsymbol{P}_{c r}, \boldsymbol{V}_{\text {lim } i t}, C_{0}\right)=0
$$

Suppose the function relationship between load $\boldsymbol{P}$ and voltage $\boldsymbol{V}$ under base case determined by $\boldsymbol{F}\left(\boldsymbol{P}, \boldsymbol{V}, C_{0}\right)$ is

$$
\boldsymbol{V}=\boldsymbol{G}(\boldsymbol{P})
$$

We can also have

$$
\boldsymbol{V}_{\text {lim } i t}=\boldsymbol{G}\left(\boldsymbol{P}_{c r}\right)
$$

Assumption The function described by (6) is decreasing function, i.e., with the increasing of $\boldsymbol{P}, \boldsymbol{V}$ is decreasing. By physical experiences it can be seen that this assumption is usually correct.

Under the above define 1, define 2, and assumption, it can be concluded that If real time operation voltage $\boldsymbol{V}>\boldsymbol{V}_{\lim i t}$, for any contingency $C_{i}(i=0,1,2, \cdots, N)$, the postcontingency operation point exists and this post-contingency operation point satisfies the inequality constraints; If real time operation voltage $\boldsymbol{V}<\boldsymbol{V}_{\lim i t}$, there at least exists one contingency $C_{I}$ such that the post-contingency operation point doesn't exist or this post-contingency operation point violates the inequality constraints.

The above method for finding bus voltage limits can be generalized to find the limits of any other operating parameters which are monotonous functions of load vector $\boldsymbol{P}$. Suppose that $r$ is some operating parameter such as line/corridor transfer power, the function relationship between $r$ and load vector $\boldsymbol{P}$ under base case is

$$
r=h(\boldsymbol{P})
$$

Case I: $r=h(\boldsymbol{P})$ is decreasing function.

Under this situation, we get a lower limit $r_{\lim i t}^{\text {lower }}$ for $r$, which is corresponding to the critical power defined in (4)

$$
r_{\lim i t}^{\text {lower }}=h\left(\boldsymbol{P}_{c r}\right)
$$

For operating under base case, $r>r_{\lim i t}^{\text {lower }}$ is considered as secure and $r<r_{\text {lim } i t}^{\text {lower }}$ is considered insecure.

Case II: $r=h(\boldsymbol{P})$ is increasing function.

Under this situation, we get a upper limit $r_{\lim i t}^{\text {upper }}$ for $r$, which is also corresponding to the critical power defined in (4)

$$
r_{\lim i t}^{u p p e r}=h\left(\boldsymbol{P}_{c r}\right)
$$

For operating under base case, $r<r_{\lim i t}^{u p p e r}$ is considered as secure and $r>r_{\lim i t}^{\text {upper }}$ is considered insecure.

In order to make the operate limits gotten more reliable, the voltage limit gotten by (7) or the limit of any other operate parameter gotten by (9) or (10) can be corrected as the following

$$
\begin{aligned}
& \boldsymbol{V}_{\lim i t}=\boldsymbol{G}\left(\boldsymbol{P}_{c r}-\Delta \boldsymbol{M}\right) \\
& r_{\lim i t}^{\text {lower }}=h\left(\boldsymbol{P}_{c r}-\Delta \boldsymbol{M}\right) \\
& r_{\text {lim } i t}^{\text {upper }}=h\left(\boldsymbol{P}_{c r}-\Delta \boldsymbol{M}\right)
\end{aligned}
$$

In (11), (12), (13), $\Delta \boldsymbol{M}$ represents imposed limit margin.

\section{Procedure for Getting Critical Buses and Critical BRANCHES}

Usually identification of critical buses and critical branches is done by modal analysis of load flow Jacobian matrix. To large power systems modal analysis is time consuming. Here we adopt a heuristic method to identify critical buses and critical branches. Voltage limits for critical buses and transfer power limits of critical branches will be visualized for monitoring.

\section{A. Critical Buses}

Fig. 6 shows PV-curves of different buses under the same contingency. The sensitivity $\Delta V_{i} / \Delta P$ of bus $i$ is used as the index to identify the critical buses under this contingency. The larger $\Delta V_{i} / \Delta P$, the more critical bus $i$ seems to be, e.g., bus 


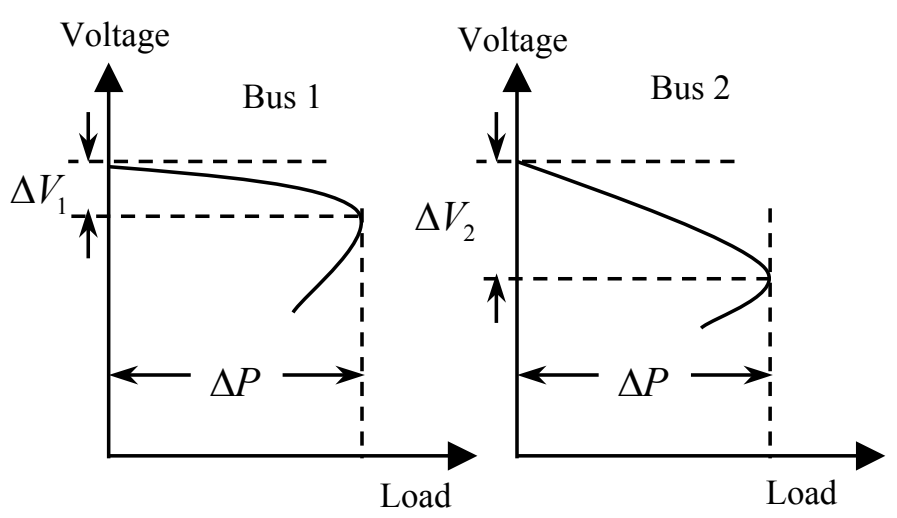

Fig. 6 PV-curves of different buses under the same contingency

2 is more critical than bus 1 . We can choose $\mathrm{M}$ buses with the largest index $\Delta V_{i} / \Delta P$ as critical buses under this contingency. Critical buses for the whole system can be gotten by integrating critical buses under each contingency among the top $\mathrm{N}$ contingencies in severity (the number $\mathrm{M}, \mathrm{N}$ can be set by users). That is

$$
C B=\cup_{i=1}^{N} C B_{i}
$$

where $C B$ is the full critical bus set for the whole system, $C B_{i}$ is the critical bus set under the $i^{\text {th }}$ contingency.

\section{B. Critical Branches}

Procedure for getting critical branches under some contingency is similar to the above procedure for getting critical buses. The index to identify the critical interfaces is $\left|\Delta P_{i, b} / \Delta P\right|$. The larger $\left|\Delta P_{i, b} / \Delta P\right|$, the more critical branch $i$ seems to be. We can choose M branches with the largest index $\left|\Delta P_{i, b} / \Delta P\right|$ as critical branches under this contingency.

Critical branches for the whole system can be gotten by integrating critical branches under each contingency among the top $\mathrm{N}$ contingencies in severity. That is

$$
C I=\cup_{i=1}^{N} C I_{i}
$$

where $C I$ is the full critical branch set for the whole system, $C I_{i}$ is the critical branch set under the $i^{\text {th }}$ contingency.

\section{NUMERICAL EXAMPLE}

The method proposed in this paper has been tested on several practical power systems. Here test results of a practical power system with 568 buses, 657 lines, 173 generators, 321 loads, 47 fixed shunts, 62 switched shunts/SVCs, 181 fixed transformers, 191 adjustable transformers are given out. In this test, load increase direction is that all loads in the whole system increase uniformly. We choose the top 10 contingencies in severity as critical contingencies, and in each contingency we find 20 critical buses and 20 critical branches. Critical buses and branches of each contingency among the top 10 severe contingencies are integrated together to form the full critical bus set and critical branch set of the whole system. Note that it's possible for one bus or branch to be critical bus or critical branch in more than one contingency. The test results include the following parts. Limited to space, here the details of the following parts are not given out.

\section{A. Top 10 Severe Contingencies}

Information about top 10 contingencies with minimal the $\mathrm{P}$ load limits are listed out, including contingency name, P-load limit value, fault information, critical buses, critical branches and some user-defined corridors. The number of selected severe contingencies can be set by users.

\section{B. Critical Buses}

After integrating 20 critical buses of each contingency among the top 10 contingency in severity, the full critical bus set is acquired. Voltage limits for these critical buses are given out. The number of selected critical buses under each contingency can be set by users.

\section{Special Monitoring Buses}

Special monitoring buses are set by users. Voltage limits of these buses will be listed out whether they are critical buses or not.

\section{Critical Branches}

After integrating critical branches of each contingency among the top 10 contingencies in severity, the full critical branch set is acquired. Branch transfer power limits are given out. The number of selected critical branches under each contingency can be set by users.

\section{E. Special Monitoring Branches}

Special monitoring branches are set by users. Branch transfer power limits of these branches will be listed out whether they are critical branches or not.

\section{F. Corridors}

Corridor transfer power limits are listed out. The number of corridors and branches each corridor includes can be set by users.

\section{CONCLUSION}

This paper gives out a method for determining power system voltage security operation limits in a practical voltage security analysis (VSA) system based on VSAT software for large power systems. These operation limits include bus voltage limits, branch/corridor transfer power limits and Pload limit of the whole system.

The voltage security operation limits are determined by the most critical contingency among the studied contingency set. The most critical contingency determines the P-load limit of the whole system, and all kinds of operation parameter limits are operation parameter values corresponding to this P-load limit under pre-contingency. An operation parameter limit is upper limit if the function relationship between this operation 
parameter and load power is an increasing curve, or lower limit if the function relationship between this operation parameter and load power is an decreasing curve. These operation parameter limits are helpful for operators to monitor the system operation state. The proposed method has been tested on several practical and it seems to work well.

In the future, voltage security control module should be included in this VSA system. That is, when power system voltage security margin is found not enough, proper control actions should be given out by VSA system. Mathematically, how to determine proper control actions comes down to a nonlinear optimization problem. Sensitivities of voltage security margin to various control parameters should be key information to determine proper control actions $[3,4]$.

\section{REFERENCES}

[1] C. W. Taylor, Power System Voltage Stability, McGraw Hill, 1994

[2] Y. Ou, and C. Singh, "Assessment of available transfer capacity and margins," IEEE Trans. Power System., Vol. 17, pp. 463-468, May 2002.

[3] R. A. Schlueter, S. Z. Liu, and K. B. Kilani, "Justification of the voltage stability security assessment and diagnostic procedure using a bifurcation subsystem method," IEEE Trans. Power System, vol. 15, pp. 1105-1111, Aug. 2000.

[4] S. Greene, I. Dobson, and F. L. Alvarado, "Sensitivity of transfer capability margins with a fast formula," IEEE Trans. Power System., Vol. 17, pp. 34-40, Feb. 2002.

[5] VSAT user manual, PowerTech Lab. Inc.., Canada, 2003

\section{BIOGRAPHIES}

N. C. Chang was born in 1977, China. He received Ph.D degree in March 2004 from Harbin Institute of Technology, Harbin, China. Now he is a research assistant in University of Hong Kong. His main research interests are power system dynamics analysis, IT application in power systems.

J. F. Su was born in 1977, China. He is now a Ph.D candidate in University of Hong Kong. His main research interests are power markets.

Z. B. Du was born in 1977, China. He is now a Ph.D candidate in University of Hong Kong. His main research interests are power system dynamic voltage stability.

L. B. Shi was born in 1971, China. He received Ph.D degree in 2000 from Chong Qing University. He is now a visiting scholar in University of Hong Kong. His main research interests are intelligence computing, IT application in power systems.

H. F. Zhou was born in 1977, China. He is now a Ph.D candidate in University of Hong Kong. His main research interests are grid computing, power markets and IT application in power systems.

Peter T. C. Tam is now a technical staff in EEE Dept. of University of Hong Kong. He received a master degree in computer science from Queensland University, Australia.

Y. X. Ni is now an associate professor in EEE Dept. of University of Hong Kong. Her main research interests are power system dynamics theory, power markets, FACTS, power electronics, IT application in power systems etc. She is a senior member of IEEE.

Felix F. Wu is now a professor in EEE Dept. of University of Hong Kong. His research interests include power markets, modern control centers, and IT applications in power systems etc. He is a fellow of IEEE. 